\title{
Oscillatory Gas Flow in a Circular Nanotube
}

\author{
M.R. King*
}

Department of Chemical Engineering, University of Rochester, Rochester, NY 14627, USA

\begin{abstract}
Gas flow through nanoscale conduits shows distinctly different physics compared to flow at the macroscale. One of the first manifestations of this is the appearance of wall slip. Here we consider the oscillatory flow of an incompressible Newtonian gas through a circular nanotube, with first order wall slip due to rarefaction. It was found that the major effect of wall slip at lower oscillation frequencies is to alter the instantaneous velocity profiles from Poiseuille-like to more plug-like, with an overall enhancement to the fluid velocity magnitude. However, at higher frequencies the enhancement to velocity magnitude due to wall slip is greatly diminished, and rarefaction introduces a region of backflow near the centerline of the nanotube. These flow characteristics may have important implications for the design of various practical applications of unsteady gas flow through nanoscale conduits, from fuel cell membranes to gas-powered nanomachines constructed from carbon nanotubes.
\end{abstract}

\section{INTRODUCTION}

Non-steady gas flow through nanotubes is important in fuel cell applications, and can occur during the synthesis of carbon nanotubes. At the nanoscale, when conduit dimensions approach the mean free path of the gas (i.e., Knudsen number $\mathrm{Kn} \rightarrow 1$ ), the physics of fluid flow deviates dramatically from the better understood macroscopic case [1]. The first manifestation of this occurs in the near-wall Knudsen layer region, where a non-zero wall slip velocity is observed. At leading order, gas flow in this regime can be modeled using the continuum Navier-Stokes equation in the bulk fluid, coupled to a first order wall-slip boundary condition at the wall. In this paper, I consider theoretically the problem of oscillatory gas flow induced by a time-periodic sinusoidal pressure gradient through a nanotube with circular crosssection. An analytical solution is obtained using the firstorder slip boundary condition, and results discussed relative to the macroscopic no-slip case. The effects of oscillation frequency and Knudsen number (via the dimensionless mean free path) are separately examined and discussed.

\section{GOVERNING EQUATIONS AND ANALYTICAL SO- LUTION}

\section{Statement of the Problem}

The macroscopic problem of oscillatory pipe flow with no-slip boundary conditions has been considered elsewhere (see, for instance, Ref. [2]). We may define the geometry as an infinitely long straight tube with circular cross-section and radius $R$. The gas is modeled as an incompressible, isothermal Newtonian fluid with constant density $\rho$ and dynamic viscosity $\mu$, a valid assumption for Mach numbers Ma $<0.3$. The gas is subjected to a periodic pressure gradient in the axial direction of the form:

$\frac{\partial p}{\partial z}=-\rho P \cos (\omega t)$

where $P$ is a constant indicating the magnitude of the pressure gradient. We seek the steady-state solution after tran-

\footnotetext{
*Address correspondence to this author at the Department of Chemical Engineering, University of Rochester, Rochester, NY 14627, USA; E-mail: mike_king@urmc.rochester.edu
}

sients have decayed away, and neglect body forces such as gravity. We further assume that the flow is unidirectional, $u_{r}=u_{\theta}=0$, and that all $\theta$-derivatives vanish due to axisymmetry. The continuity equation then simplifies to:

$$
\frac{\partial u_{z}}{\partial z}=0
$$

which implies that the one non-zero velocity component is only a function of $r$ and $t, u_{z}=u_{z}(r, t)$. The simplified form of the $r$-component of the Navier-Stokes equation:

$0=\frac{\partial p}{\partial r}$

indicates that the pressure will only vary axially and with time, $p=p(z, t)$.

The $z$-component of the Navier-Stokes equation yields the main governing equation for $u_{z}$ :

$\frac{\partial u_{z}}{\partial t}=P \cos (\omega t)+\mu\left(\frac{\partial^{2} u_{z}}{\partial r^{2}}+\frac{1}{r} \frac{\partial u_{z}}{\partial r}\right)$

The appropriate boundary condition for $O(\mathrm{Kn})$ wall slip will be introduced later. To obtain a general solution to this differential equation, we use the complex notation:

$-\frac{1}{\rho} \frac{\partial p}{\partial z}=P e^{i \omega t}$

where only the real part has physical significance. The complex notation suggests a velocity distribution of the following form:

$u_{z}(r, t)=f(r) e^{i \omega t}$

where $f(r)$ is an unknown function to be determined. Substitution of this form into the governing equation yields an ordinary differential equation for $f(r)$ :

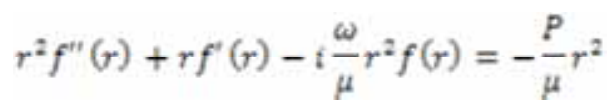

This is recognized as an inhomogeneous Bessel's equation of the zeroth order. The particular solution producing the term on the right hand side is found to be simply: 
$f_{p}(r)=-i \frac{\mu}{\omega}$

The general solution to the homogeneous Bessel equation is the following linear combination:

$f_{h}(r)=C_{1} J_{0}\left(\sqrt{\frac{-i \omega}{\mu} r}\right)+C_{2} Y_{0}\left(\sqrt{\frac{-i \omega}{\mu}} r\right)$

where $J_{0}$ is the Bessel function of the first kind and zeroth order:

$10\left(\sqrt{\frac{-i \omega}{\mu} r}\right)=\sum_{n=0}^{\infty} \frac{(-1)^{n}}{(n !)^{2}}\left(\sqrt{\frac{-i \omega}{\mu}} \frac{r}{2}\right)^{2 n}$

and $Y_{0}$ is the Bessel function of the second kind and zeroth order:

$$
\begin{aligned}
Y_{0}(z)=\frac{2}{\pi} J_{0}(z) & \left(\ln \left(\frac{z}{2}+\gamma\right)\right. \\
= & -\frac{2}{\pi} \sum_{n=0}^{=} \frac{(-1)^{n}}{(n !)^{2}}\left(\frac{z}{2}\right)^{2 n}\left(\frac{1}{n}+\frac{1}{n-1}\right. \\
& +\cdots+1)
\end{aligned}
$$

The constant $\gamma \approx 0.5772$ is known as the EulerMáscheroni constant. The Bessel function of the second kind $Y_{0}(\sqrt{-i \omega / \mu} r)$ has a logarithmic singularity at $r=0$, so to enforce the condition that the velocity remain bounded at the center of the nanotube we set $C_{2}=0$. Thus, the velocity solution to within an integration constant is:

$u_{z}(r, t)=\left[C_{1} J_{0}\left(\sqrt{\frac{-i \omega}{\mu}} r\right)-i \frac{P}{\omega}\right] e^{i \omega t}$

\section{First-Order Wall Slip Boundary Condition}

There is a region near the tube wall in which the gas is out of equilibrium, called the Knudsen layer. The Knudsen layer has a thickness on the order of the mean free path of the gas. When the relevant dimensions of a flow conduit approach the length scale of the Knudsen layer thickness, typically at the nanoscale, interesting behavior emerges. The transition between macroscopic and nanoscale flow regimes is best expressed in terms of the dimensionless Knudsen number, defined as $\mathrm{Kn}=\lambda / L$ where $\lambda$ is the mean free path of the gas and $L$ is the characteristic length of the control volume. The mean free path $\lambda$ can be thought of as the average distance a gas molecule travels between molecular collisions. Thus, when the frequency of molecular collisions with the wall approaches the frequency of intermolecular gas collisions, the fluid ceases to behave as a continuum. For reference, the mean free path of air at standard temperature and pressure is $\lambda_{0}=49 \mathrm{~nm}$. Various theoretical estimates of the mean free path exist, depending on the collision model assumed [1].

The slip flow regime, as we will consider here, occurs in the approximate range of $10^{-3}<\mathrm{Kn}<10^{-1}$. Within this parameter range, the complex physics in the Knudsen layer can be well approximated by replacing the common no-slip boundary condition with a velocity slip at the wall. It has been known for quite some time that the flow of rarified gas results in slipping at the walls [3]. If $s$ denotes the direction parallel to the wall, the slip velocity may be expressed as:

$u_{\text {slip }}=u_{z}-u_{\text {wall }}=\left.\xi \frac{\partial u_{s}}{\partial n}\right|_{w}$

where $\xi$ is a length called the "coefficient of slip" and $n$ is the normal direction. Maxwell estimated the magnitude of the coefficient of slip from the kinetic theory of gases to be [4]:

$\xi=\frac{2-\sigma}{\sigma} \lambda$

where $\sigma$ represents the fraction of molecules that are absorbed by the surface due to wall roughness or due to a condensation-evaporation process. These molecules are then reemitted with velocities corresponding to those of quiescent gas at the temperature of the wall. The remaining fraction of molecules, $1-\sigma$, are perfectly reflected by the wall and retain their original momentum. In non-isothermal systems there also exists an additional $O\left(\mathrm{Kn}^{2}\right)$ slip term due to thermal gradient in the $s$-direction.

\section{Analytic Solution for Gas Velocity}

Thus, for our cylindrical geometry, the appropriate slip boundary condition takes the form:

$\left.u_{z}\right|_{r=R}=\left.\frac{2-\sigma}{\sigma} \lambda \frac{\partial u_{z}}{\partial r}\right|_{r=R}$

Making use of the following two Bessel function identities:

$\frac{d}{d x}\left[x^{m} j_{m}(x)\right]=x^{m} j_{m-1}(x)$

$J_{-m}(x)=(-1)^{m} J_{m}(x)$

we can enforce the wall slip boundary condition as:

$$
\begin{aligned}
\frac{\sigma-2}{\sigma} \lambda\left[C_{1} \sqrt{\frac{-i \omega}{\mu}} J_{1}\left(\sqrt{\frac{-i \omega}{\mu}} R\right)\right] e^{i \omega t} \\
=\left[C_{1} J_{0}\left(\sqrt{\frac{-i \omega}{\mu}} R\right)-i \frac{P}{\omega}\right] e^{i \omega t}
\end{aligned}
$$

This yields the constant:

$$
\begin{array}{r}
C_{1}=i P / \omega\left[J_{0}\left(\sqrt{\frac{-i \omega}{\mu}} R\right)\right. \\
\left.-\frac{\sigma-2}{\sigma} \lambda \sqrt{\frac{-i \omega}{\mu}} J_{1}\left(\sqrt{\frac{-i \omega}{\mu}} R\right)\right]^{-1}
\end{array}
$$

For convenience we define the following two parameters:

$\alpha=\sqrt{\omega / \mu} R$

$\beta=\frac{\sigma-2}{\sigma} \lambda \sqrt{\frac{\omega}{\mu}}$

where $\alpha$ represents a dimensionless frequency of oscillation, and $\beta$ represents the relative importance of rarefaction. In terms of these new parameters, the flow solution becomes: 

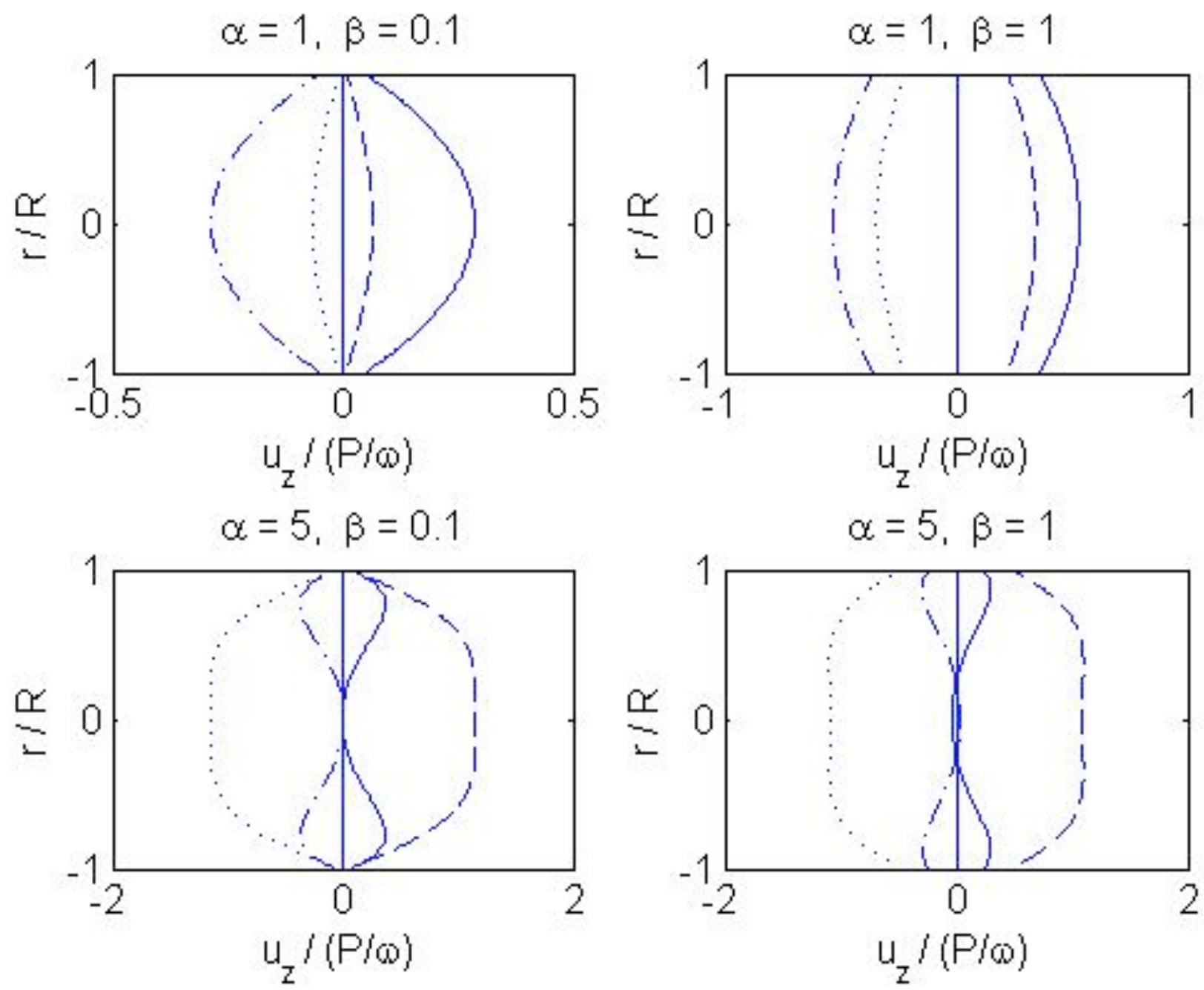

Fig. (1). Plot of the instantaneous gas velocity for different values of $\alpha$ and $\beta$. The dimensionless times $\omega t$ correspond to: 0 (solid line), $\pi / 2$ (dashed line), $\pi$ (dash-dot), and $3 \pi / 2$ (dotted line) in each subfigure.

$$
\begin{aligned}
u_{z}(r, t)=\frac{P}{\omega} \operatorname{Real} & \left\{-i e^{i \omega t}[1\right. \\
& \left.\left.-\frac{J_{0}(\sqrt{-i} \alpha r / R)}{J_{0}(\sqrt{-i} \alpha)-\sqrt{-i} \beta J_{1}(\sqrt{-i} \alpha)}\right]\right\}
\end{aligned}
$$

where we have taken the real part to extract the physically meaningful velocity component.

\section{RESULTS AND DISCUSSION}

Fig. (1) shows plots of the instantaneous velocity profile for different values of the two dimensionless parameters $\alpha$ and $\beta$. A number of interesting predictions are evident. At lower frequency and Knudsen number $(\alpha=1, \beta=0.1)$, the instantaneous velocity profiles look similar to the macroscopic Poiseuille flow. The gas flow is in phase with the imposed pressure gradient. At low frequency and higher Knudsen number $(\alpha=1, \beta=1)$, the flow appears more plug-like. Note that the magnitude of flow velocity is increased relative to the no-slip case, due to reduced flow resistance in the near wall region. At higher oscillation frequency, the instantaneous velocity profiles look quite different from the steady Poiseuille flow. At $\alpha=5$ and $\beta=1$ the fluid towards the center of the nanotube experiences a phase lag of $\pi / 2$ behind the imposed pressure gradient. Finally, at higher oscillation frequency and higher relative rarefaction $(\alpha=5, \beta=1)$, we see that at $\omega t=0, \pi$ the velocity at the center of the nanotube is remarkably predicted to be in the opposite direction of the bulk flow, a phenomenon that is not observed at comparable oscillation frequencies in the absence of wall slip. Additionally, it is important to note that unlike the lower frequency case, here the presence of rarefaction-induced wall slip affords no significant increase in the magnitude of the peak velocity.

\section{CONCLUSIONS}

In this paper, I have considered the problem of oscillatory gas flow through a circular nanotube subject to firstorder slip boundary conditions. Interestingly, it was found that at lower oscillation frequencies the effect of wall slip is to increase the magnitude of gas velocity, however at higher frequencies this effect of rarefaction is greatly attenuated. Such frequency response could have important implications for nanoscale devices that are driven by oscillatory gas flow, such as the nanoscale engine comprised of a carbon nanotube oscillator, motor, channel, and nozzle, considered by Kang and Hwang [5]. In applications such as these, where device efficiency depends on the pulsatile rate of gas flow through 
nanoscale conduits, the device dimensions and operating parameters should be carefully chosen to minimize flow resistance while possibly eliminating backflow in the center region as demonstrated in Fig. (1) $(\alpha=5, \beta=1)$ that could limit the forward stroke of pulsatile fluidic actuation or energy generation.

\section{REFERENCES}

[1] Colin, S. Single-phase gas flow in microchannels. In Heat Transfer and Fluid Flow in Minichannels and Microchannels, by Kandlikar, S.G.; Garimella, S.; Li, D.; Colin, S.; King, M.R. Elsevier Science, Amsterdam, 2006.

[2] Spurk, J.H. Fluid Mechanics. Problems and Solutions. Springer, Berlin, 1997.

[3] Kennard, E.H. Kinetic Theory of Gases. McGraw-Hill Book Company, New York, 1938.

[4] Maxwell, J.C. Philos. Trans. Royal Soc., 1879, 170, 231.

[5] Kang, J.W.; Hwang, H.J. Nanotechnology, 2004, 15, 1633. 